\title{
IDENTIFICAÇÃO E VALORAÇÃO DOS CRITÉRIOS DE DECISÃO EM PROJETOS DE DESCOMISSIONAMENTO OFFSHORE
}

\author{
IDENTIFICATION AND VALUATION OF DECISION CRITERIA IN OFFSHORE \\ DECOMMISSIONING PROJECTS
}

Yarly Queiroz de Lima Mestrando em Administração de Empresas - Ibmec RJ Petróleo Brasileiro S.A. - Petrobras. Rio de Janeiro, RJ - Brasil. engyarly@gmail.com

\author{
Luiz Flávio Autran Monteiro Gomes \\ Doctor of Philosophy in Engineering pela University of California \\ Centro Universitário Ibmec - Ibmec RJ \\ Rio de Janeiro, RJ - Brasil \\ luiz.gomes@professores.ibmec.edu.br
}

\begin{abstract}
Resumo
O cenário de volatilidade de preços do barril de petróleo causado pela pandemia COVID-19 ainda não é totalmente conhecido por ainda estarmos vivenciando os impactos da abrupta alteração da demanda. Entre os inúmeros impactos, fatores macro e microeconômicos podem antecipar o processo de descomissionamento de unidades de exploração e produção de petróleo e gás natural. O objetivo deste estudo foi explorar a ordem de preferências de critérios e subcritérios aplicáveis a métodos de Apoio Multicritério à Decisão na seleção de alternativas de descomissionamento de unidades marítimas de exploração e produção de petróleo e gás natural. Para alcançar o objetivo proposto realizou-se pesquisa de campo com membros de equipe de projetos de descomissionamento. Aplicou-se os resultados de preferência entre critérios e subcritérios no descomissionamento da plataforma Alpha Jacket do campo de Brent. Constatou-se o alinhamento das preferências a políticas de segurança, preservação da vida e do meio ambiente, aderente aos instrumentos de incentivo de órgãos reguladores, bem como aos valores éticos e pilares estratégicos da empresa em que estão inseridos.
\end{abstract}

Palavras-chave: Descomissionamento. Óleo e gás. Apoio multicritério à decisão.

\begin{abstract}
The scenario of volatility in the price of a barrel of oil caused by the pandemic COVID-19 is not yet fully known because we are still experiencing the impacts of the abrupt change in demand. Among the countless impacts, macro and microeconomic factors can anticipate the decommissioning process of oil and natural gas exploration and production units. The objective of this study was to explore the order of preferences of criteria and sub-criteria applicable to methods of Multiple Criteria Decision Aid in selection of decommissioning alternatives for offshore oil and natural gas exploration and production units. In order to achieve the proposed objective, field research was carried out with team members of decommissioning projects. The results of preference between criteria and sub-criteria were applied in the decommissioning of the Alpha Jacket platform in the Brent field. There was an alignment of preferences with safety policies, preserving life and the environment, adhering to the incentive instruments of regulatory bodies, as well as the ethical values and strategic pillars of the company in which they operate.
\end{abstract}

Keywords: Decommissioning. Oil and gas. Multiple criteria decision aid.

Cite como

American Psychological Association (APA)

Lima, Y. Q., \& Gomes, L. F. A. M. (2021, maio/ago.). Identificação e valoração dos critérios de decisão em projetos de descomissionamento offshore. Revista de Gestão e Projetos (GeP), 12(2), 9-27. https://doi.org/10.5585/gep.v12i2.19781. 


\section{Introdução}

Entende-se por descomissionamento o conjunto de atividades associadas à interrupção definitiva da operação das instalações, ao abandono permanente e arrasamento de poços, à remoção de instalações, à destinação adequada de materiais, resíduos e rejeitos e à recuperação ambiental da área (ANP, 2020)

O descomissionamento de instalações de exploração e produção de petróleo e gás natural (oil and gas - O\&G) tem sido tema de estudo de muitos autores (Bull \& Love, 2019; Eke et al., 2020; Ekins et al., 2006; Martins et al., 2020) bem como uma preocupação relevante de diversas empresas do setor (Repsol, 2017; Shell, 2017b). A aplicação de métodos multicritérios de apoio à tomada de decisão também pode ser vista em uma quantidade significativa de estudos (Fowler et al., 2014; Henrion et al., 2015; Marfatia, 2019; Martins et al., 2020).

O descomissionamento é a última etapa no ciclo de vida de um projeto de exploração e produção de O\&G. Deve ser iniciado quando se perde a eficiência econômica do reservatório, ou seja, quando os custos de produção superam as receitas, provocados pela queda do preço do petróleo, obsolescência de equipamentos, tecnologia ou declínio do fator de produção do reservatório após anos de extração.

No mundo há um número crescente de plataformas marítimas atingindo o fim de sua vida útil devido à quantidade de campos offshore de petróleo e gás que foram desenvolvidos nas últimas décadas. No ano de 2018 totalizaram 1.332 plataformas de produção de petróleo no mar, destas 51 localizadas na costa brasileira (Statista, 2018). A indústria do descomissionamento está em rápida expansão e deve gerar bilhões em receitas e criar milhares de empregos em todo o mundo (Marfatia, 2019).

De acordo com levantamentos da Agência Nacional do Petróleo, Gás Natural e Biocombustíveis (ANP) estima-se que nos próximos 5 anos, somente no Brasil, sejam investidos mais de 38 bilhões de reais em projetos de descomissionamento que vão desde retirada de equipamentos e recuperação de áreas até o arrasamento e abandono de mais de 1.100 poços de produção de O\&G, (ANP, 2021).

O processo de descomissionamento é controverso pois envolve, em cada alternativa, diferentes níveis de custos, benefícios e riscos para grupos distintos de partes interessadas e para o meio ambiente (Schroeder \& Love, 2004). Keeney e Raiffa (1993) afirmam que em um objetivo há três características: um contexto de decisão, um objeto e uma direção de preferência. No presente estudo efetuou-se uma pesquisa com 15 indivíduos, membros de equipe de projetos de descomissionamento de uma companhia de óleo e gás, a fim de conhecer a estrutura de preferência entre critérios e subcritérios utilizados na avaliação e seleção de alternativas ao descomissionamento de unidades de produção de O\&G offshore. Agrupou-se as ordens de preferência por experiência em projetos de 
descomissionamento e as ponderações foram obtidas por meio da aplicação da técnica ROC (Rank Order Centroid), do método Simple Multi-attribute Rating Technique Exploiting Ranks - SMARTER (Edwards \& Barron, 1994). Aplicou-se as ponderações obtidas nos dados de avaliação das alternativas de descomissionamento da plataforma Alpha Jacket do campo de Brent (Shell, 2017a, 2017b).

Conhecer as preferências dos membros das equipes de projeto, bem como seus julgamentos de valor permite direcionar os esforços em novas abordagens e estratégias de comunicação, disseminação do conhecimento e aprendizagem organizacional. Os entrevistados trabalham na identificação e desenvolvimento de alternativas, elaboram análises de disciplinas técnicas, bem como conteúdo e informações relevantes ao suporte a tomada de decisão de qual projeto será adotado como solução para o descomissionamento.

\section{Referencial teórico}

$\mathrm{Na}$ seção a seguir, apresentam-se os principais referenciais teóricos no desenvolvimento de alternativas ao descomissionamento de ativos de produção de O\&G offshore, seguidos das mais relevantes abordagens do apoio multicritério à decisão.

\subsection{Descomissionamento de unidades de produção offshore}

Para a Oil and Gas United Kingdom (OGUK, 2020), o impacto total da pandemia COVID-19 na volatilidade dos preços e demandas das commodities ainda está para ser visto. A recente e brusca queda nos preços do petróleo (Investing, 2021), em sua maior parte provocada pela vertiginosa queda na demanda, pode antecipar o limite econômico, conforme demonstra a Figura 1. Trata-se de um cenário que coloca ainda mais pressão no fluxo de caixa das empresas exploradoras de O\&G. Por outro lado, a redução de custos operacionais pode beneficiar o negócio, tornando a produção viável economicamente por mais tempo. 
Figura 1 - Representação do ciclo de vida de projeto de $O \& G$ por limite econômico

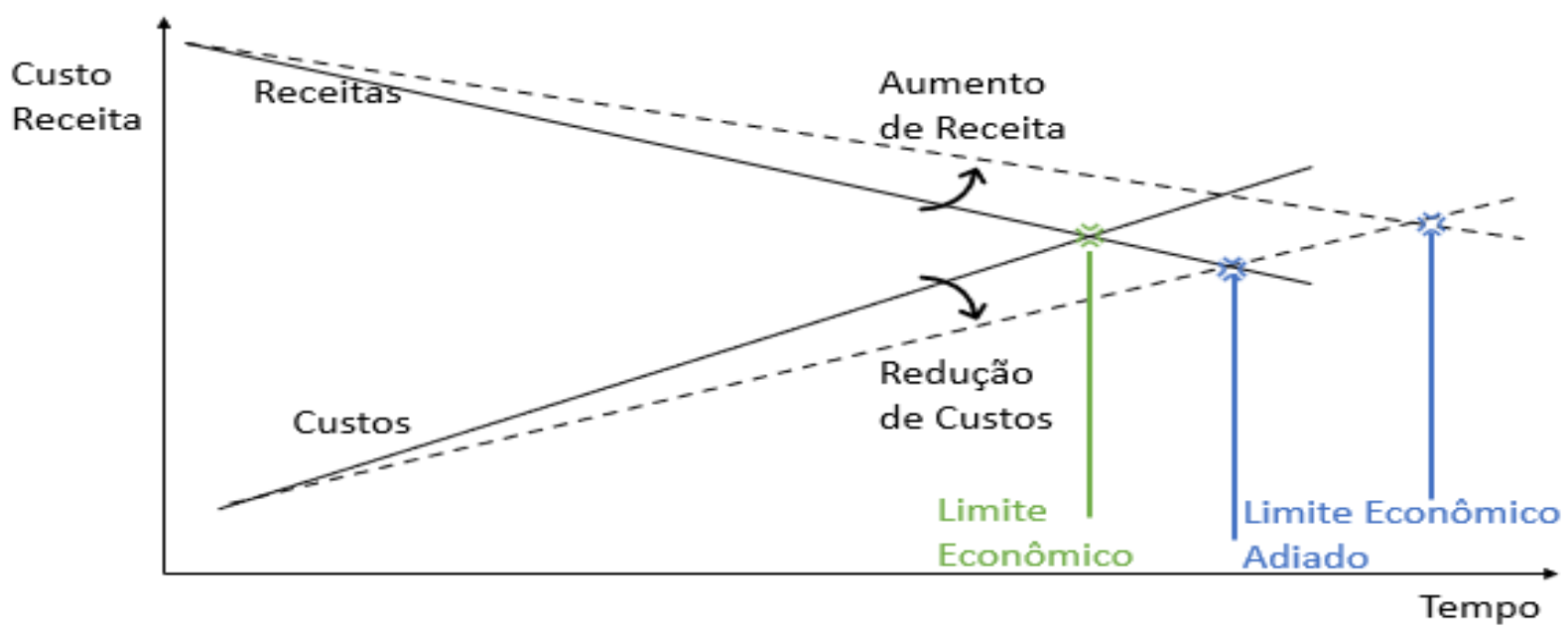

Fonte: Kaiser (2019).

A preocupação com as atividades de exploração de recursos do fundo marítimo está presente desde a Convenção das Nações Unidas na Plataforma Continental (UN, 1958) e o Direito do Mar (UN, 1995). Especificamente sobre o descomissionamento tratam que todas as instalações abandonadas ou em desuso devem ser inteiramente removidas, não devendo resultar em qualquer interferência injustificável na navegação, pesca ou na conservação dos recursos vivos do mar (UN, 1958).

A ANP através da resolução $\mathrm{N}^{\circ} 817$, de 24 de abril de 2020, trata o descomissionamento como sendo o conjunto de atividades associadas à interrupção definitiva da operação das instalações, ao abandono permanente e arrasamento de poços, à remoção de instalações, à destinação adequada de materiais, resíduos e rejeitos e à recuperação ambiental da área.

Diferentes opções de descomissionamento podem ser aplicadas aos diversos componentes de um ativo de produção offshore. Eke et al.(2020) afirmam que os principais componentes considerados durante o processo de descomissionamento incluem os poços de produção, instalações de superfície e instalações submarinas, conforme Figura 2. 
Figura 2 - Tipos de plataformas e dimensão de lâmina d'água por tipo de unidade de produção offshore de O\&G

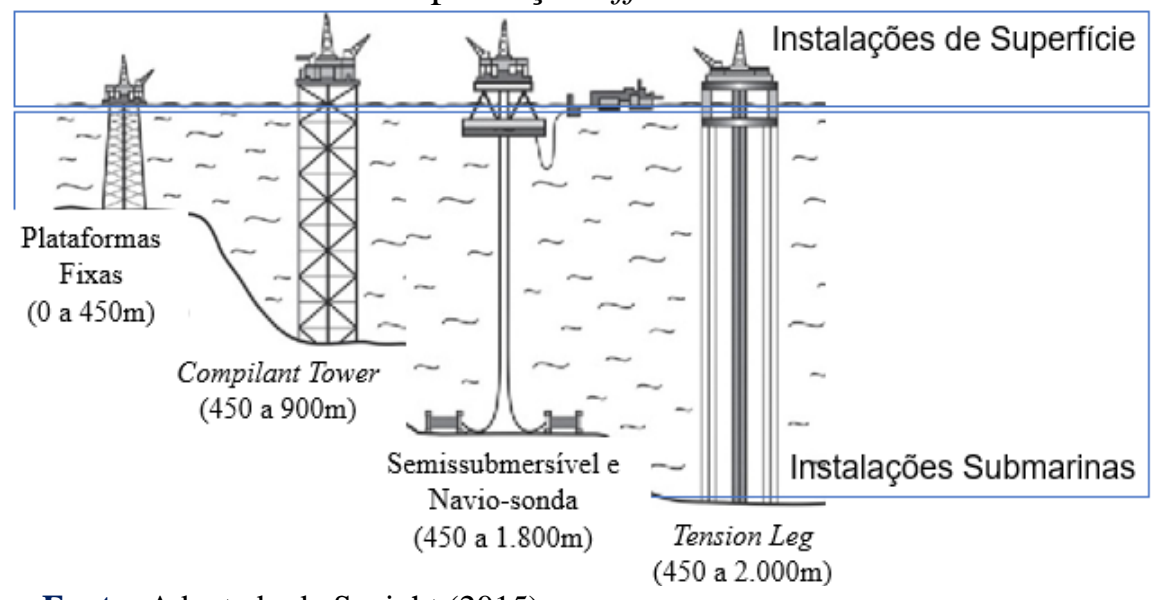

Fonte: Adaptado de Speight (2015).

Plataformas de produção offshore são responsáveis pela maior parcela de extração de O\&G no Brasil, sendo apenas a camada pré-sal responsável pelo aumento de $54 \%$ no período entre setembro de 2019 e setembro de 2020
(ANP, 2020a). No campo de desenvolvimento de alternativas ao descomissionamento Fowler et al. (2014) resume as opções de remoção, remoção parcial e abandono em formato de árvore de decisão conforme Figura 3.

Figura 3 - Alternativas de descomissionamento de unidade de produção offshore

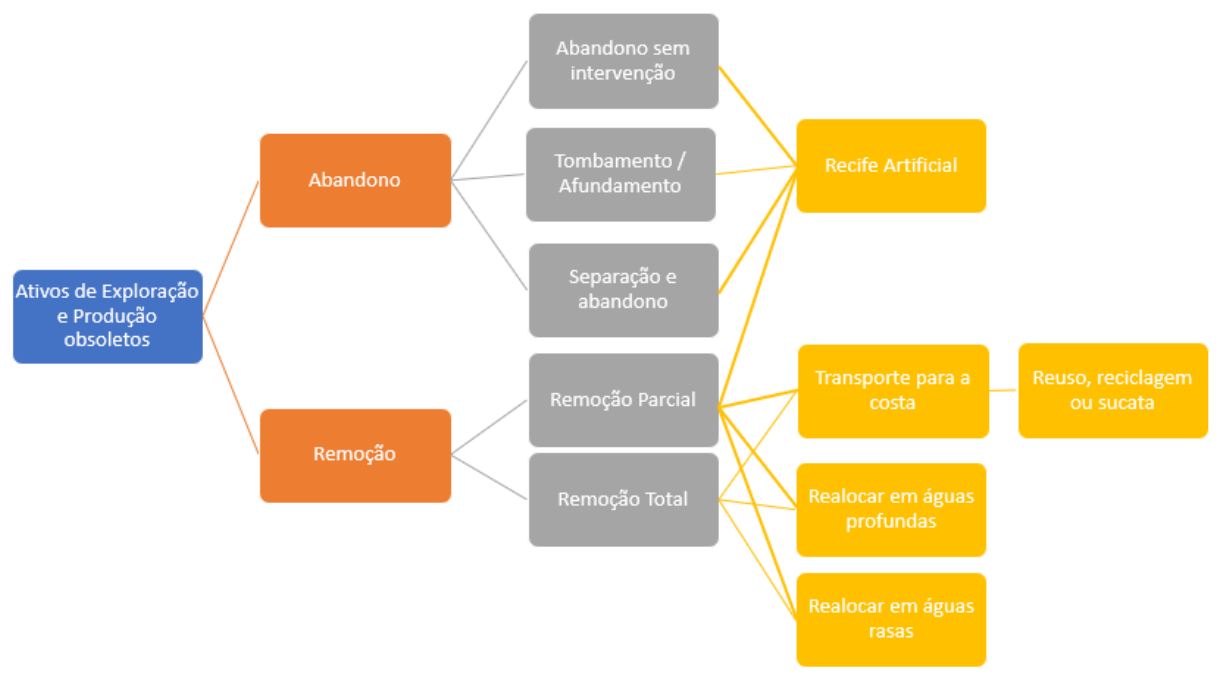

Fonte: Adaptado de Fowler et al. (2014)

A lista de opções de descomissionamento pode ser modificada para se adequar ao cenário de descomissionamento específico, sem afetar as etapas subsequentes no processo de decisão
(Fowler et al., 2014). Instalações de superfície têm sido reaproveitadas em novos desenvolvimentos de petróleo, mas também existem oportunidades tangíveis de 
reaproveitamento para uso em novas indústrias de CCUS (Carbon Capture, Utilisation and Storage), hidrogênio e geração de energia eólica offshore (IEA, 2020; OGUK, 2020).

Martins et al.(2020) apresentam levantamento de alternativas mais detalhadas, segregando as opções entre estrutura de superfície e estrutura submarina, abrangendo desde a criação de unidades de geração eólica ou estações de estudos oceanográficos até opções de remoção de dutos submarinos flexíveis e rígidos. A Figura 4 apresenta uma árvore de decisão ampliada.

Figura 4 - Alternativas de descomissionamento de unidade de produção offshore

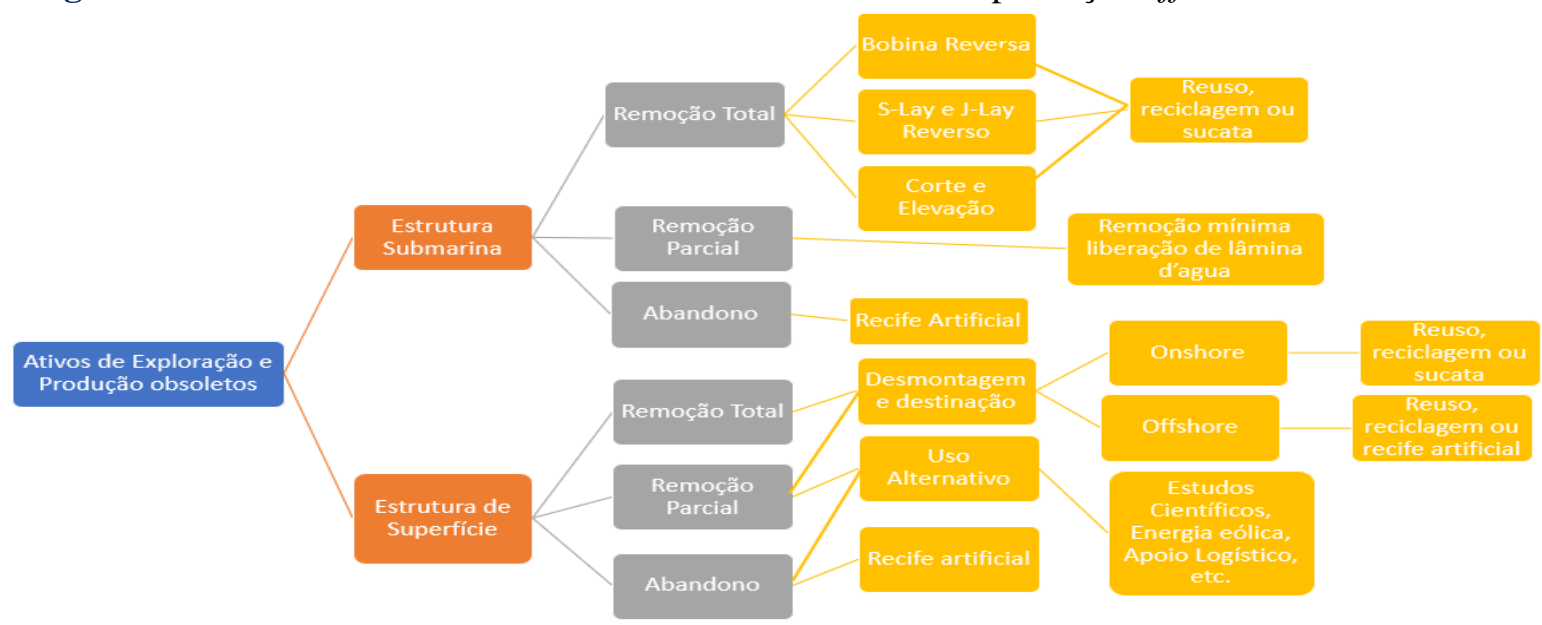

Fonte: Adaptado de Martins et al. (2020)

As

alternativas

para descomissionamento são amplas e novas soluções ou tecnologias de reaproveitamento podem surgir. Em linhas gerais as decisões relativas às alternativas de descomissionamento devem ser estudadas caso a caso (Schroeder \& Love, 2004).

\subsection{O enfoque multicritério para a tomada de decisão}

O principal objetivo do Apoio Multicritério à Decisão (AMD) é fornecer um conjunto de metodologias de agregação de critérios que permitam o desenvolvimento de modelos de suporte à decisão (Doumpos \&
Zopounidis, 2002). Tomadores de decisão buscam ajuda em um AMD exatamente porque têm dificuldade em entender o que realmente querem fazer e quais opções melhor atenderão às aspirações no longo prazo (Belton \& Stewart, 2002)

Martins et al. (2020) realizaram extenso levantamento bibliográfico de artigos e relatórios técnicos que utilizaram métodos de AMD em projetos de descomissionamento em diversos segmentos industriais (energia nuclear, eólica, mineração, $\mathrm{O} \& \mathrm{G}, \quad$ logística). $\mathrm{O}$ quantitativo por método AMD do estudo bibliográfico pode ser observado na Tabela 1. 
Tabela 1 - Artigos científicos e relatórios técnicos que utilizaram métodos AMD em projetos de descomissionamento

\begin{tabular}{lcccccc}
\hline & Método AMD & AHP $^{1}$ & PROMETHEE $^{2}$ & SAW $^{3}$ & $\begin{array}{c}\text { Avaliações } \\
\text { Comparativas }^{4}\end{array}$ & Outros \\
\hline Todos & $45^{5}$ & 16 & 6 & 4 & 10 & 18 \\
O\&G & 14 & 3 & 0 & 1 & 9 & 1 \\
\hline
\end{tabular}

Nota. 1AHP - Analytic Hierarchy Process (Saaty, 2008). 2PROMETHEE - Preference Ranking Organization Method. 3SAW - Simple Additive Weighting. 4Métodos derivados do Guia OGUK (2015). 5Contempla estudos que utilizaram mais de um método AMD.

Fonte: Adaptado de Martins et al. (2020).

Apesar da forte presença do método AHP, $35 \%$ dos estudos de descomissionamento já realizados, ao segmentar os descomissionamentos destinados à indústria de O\&G percebe-se forte relevância de métodos de Avaliações Comparativas (Repsol, 2017; Shell, 2017b). Estes estudos provêm essencialmente de derivações do Guidelines for Comparative Assessment in Decommissioning Programm (OGUK, 2015).

Independentemente do método utilizado, para que um agente de decisão possa efetuar uma escolha sobre um conjunto de ações potenciais, supõe-se que uma análise será balanceada em diferentes eixos de avaliação (GOMES e GOMES, 2014). A resolução ANP $\mathrm{N}^{\circ} 817$ cita cinco eixos, ou critérios, que devem ser adotados na elaboração do Programa de Descomissionamento de Instalações, são eles: técnico, ambiental, social, econômico e de segurança. No entanto, estes mesmos critérios já foram considerados em outras avaliações e escolhas de alternativas ao descomissionamento de unidade de produção $O \& G$, tanto em relatórios técnicos (Repsol, 2017; Shell, 2017b), quanto em estudos científicos (Eke et al., 2020; Ekins et al., 2006; Fowler et al., 2014; Martins et al., 2020). Como os critérios são abrangentes é corriqueiro adotar-se subcritérios que avaliem cada projeto de descomissionamento de forma particular e que melhor reflitam as preferências do decisor. A escolha dos subcritérios para o estudo em análise será discutida no item a seguir.

\section{Metodologia}

A metodologia de pesquisa adotada neste artigo é de natureza exploratória, descritiva e aplicada, quanto aos fins, e pode-se classificar como pesquisa de campo quanto aos meios. Para Vergara (2016) uma pesquisa exploratória é realizada em área na qual há pouco conhecimento acumulado e sistematizado. A recente publicação da resolução ANP No 817 introduz novos conceitos e reformulações na regulação de descomissionamento de unidade de O\&G. O aspecto descritivo da pesquisa revela-se ao trazer à tona as preferências entre critérios e subcritérios de membros de equipes de projeto de descomissionamento.

\section{Através do Microsoft Forms ${ }^{\circledR}$} submeteu-se um formulário de pesquisa a 15 indivíduos. Todos compõem uma equipe de projetos de descomissionamento de uma 
companhia de óleo e gás e estão envolvidos na elaboração de documentação técnica que suporta o processo de tomada de decisão na seleção de alternativas ao descomissionamento de unidades de produção de O\&G offshore. No formulário de pesquisa segregou-se a experiência por tempo profissional na indústria de O\&G bem como com projetos de descomissionamento.

De posse dos formulários de pesquisa respondidos, segregou-se por tempo de experiência e autopercepção em projetos de descomissionamento. Do ordenamento de preferências entre critérios e subcritérios obteve-se as ponderações por meio da aplicação da técnica ROC (Rank Order Centroid), do método SMARTER (Edwards \& Barron, 1994). O caráter aplicado da pesquisa revela-se quando a ponderação foi adotada no caso de descomissionamento da plataforma Alpha Jacket apresentado no relatório Shell (2017a) e discutidos seus efeitos na avaliação dos resultados.

\subsection{Seleção de critérios e subcritérios}

Para Kueppers et al. (2004) a parte mais importante do processo de tomada de decisão é a elaboração da lista de critérios e subcritérios. É sob a perspectiva dos resultados de cada critério, seu impacto na escolha, que cada alternativa será avaliada. Belton e Stewart (2002) complementam que é fundamental para qualquer análise de problemas de decisão de múltiplos critérios a importância relativa a ser atribuída a cada um dos critérios.

Os critérios submetidos a avaliação de preferência dos entrevistados são mandatórios, constam na resolução ANP No 817 e, portanto, todo projeto de descomissionamento no Brasil está enquadrado na regulação em epígrafe. Já para os subcritérios, adotou-se o levantamento feito por Martins et al. (2020), excluindo os não aplicáveis à realidade brasileira ou inadequados aos projetos que constam na carteira da equipe envolvida, Tabela 2.

Tabela 2 - Critérios e subcritérios submetidos aos entrevistados

Continua

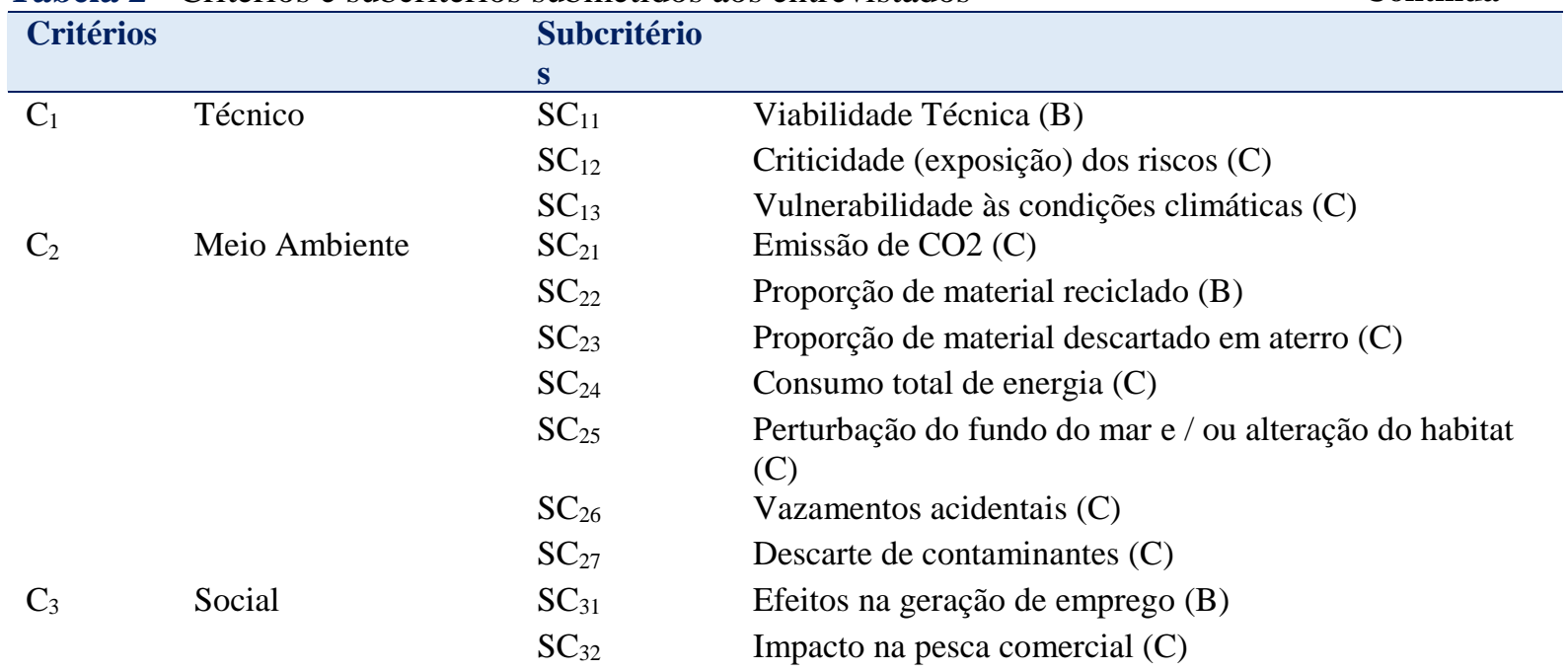




$\begin{array}{llll} & & & \text { Conclusão } \\ & & \mathrm{SC}_{33} & \text { Impacto nas comunidades }(\mathrm{C}) \\ \mathrm{C}_{4} & \text { Econômico } & \mathrm{SC}_{34} & \text { Impacto residual na navegação local }(\mathrm{C}) \\ & \mathrm{SC}_{41} & \text { Custo total }(\mathrm{C}) \\ \mathrm{C}_{5} & \mathrm{SC}_{42} & \text { Incertezas e riscos de custo }(\mathrm{C}) \\ & \text { Segurança } & \mathrm{SC}_{51} & \text { Exposição a risco da equipe de projeto onshore }(\mathrm{C}) \\ & & \\ & \text { Operacional } & \mathrm{SC}_{52} & \text { Exposição a risco da equipe de projeto offshore }(\mathrm{C}) \\ & \mathrm{SC}_{53} & \text { Exposição a riscos dos usuários das vias marinhas }(\mathrm{C})\end{array}$

Nota. (B) Subcritério tipo benefício; (C) Subcritério tipo custo.

Fonte: Adaptado de Martins et al. (2020)

\subsection{Ponderação dos critérios e subcritérios através do método SMARTER}

Edwards e Barron (1994) propuseram o emprego de uma técnica simples de modelagem e atribuição de pesos na representação das preferências dos decisores, o ROC (Rank Order Centroid). Adotou-se esta técnica, para a definição dos pesos dos critérios e subcritérios. Sendo $W_{n}$ o peso do critério $C_{n}$ qualquer, para $\mathrm{c}=1, \ldots, \mathrm{n}$, admite-se que se $W_{1}>W_{2}>\cdots>$ $W_{n}$, portanto:

$$
\begin{aligned}
& W_{1}=\left(1+\frac{1}{2}+\frac{1}{3}+\cdots \frac{1}{n}\right) / n \\
& W_{2}=\left(0+\frac{1}{2}+\frac{1}{3}+\cdots \frac{1}{n}\right) / n \\
& W_{n}=\left(0+\cdots+0+\frac{1}{n}\right) / n
\end{aligned}
$$

Generalizando:

$$
W_{n}=\left(\frac{1}{n}\right) \sum_{i=n}^{n} \frac{1}{i}
$$

Além da simplicidade da aplicação e uso da técnica ROC, Barron e Barrett (1996) confirmaram sua eficácia com extensos testes que levaram a melhores escolhas das alternativas em até $87 \%$ das vezes e evidenciaram perdas de valores da utilidade global menor que $2 \%$.

\section{Análise e discussão dos resultados}

\subsection{Preferência entre critérios e subcritérios}

Através dos formulários de entrevistas obteve-se as ordens de preferência entre os critérios e subcritérios de cada entrevistado. Do 
resultado agregado obteve-se as ordens de

preferência $\mathrm{C}_{5}>\mathrm{C}_{2}>\mathrm{C}_{1}>\mathrm{C}_{4}>\mathrm{C}_{3}$. A Figura

5 sintetiza a frequência de resultados.

Figura 5 - Radar de frequência de posição dos critérios de avaliação das alternativas

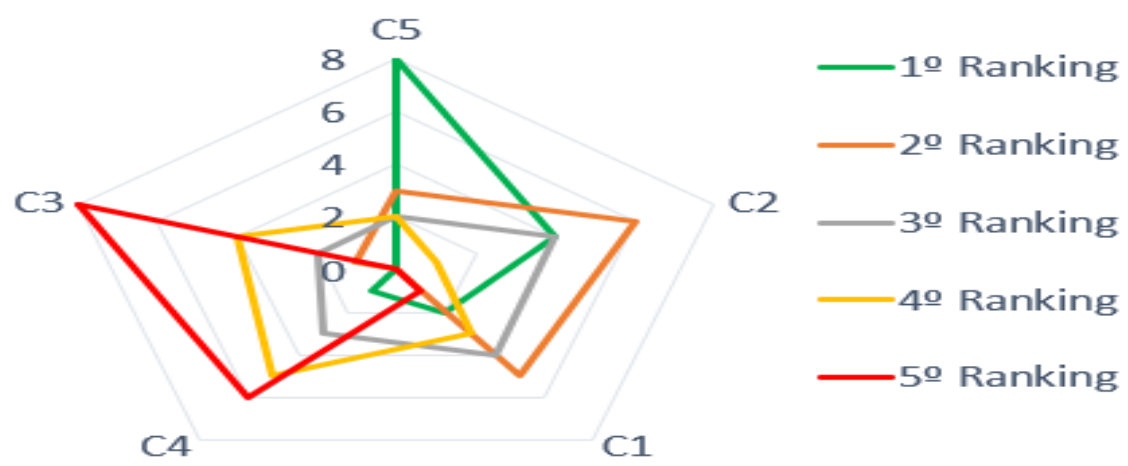

Fonte: Elaboração própria

Dos 15 profissionais entrevistados 8 possuem menos de 1 ano trabalhando com projetos de descomissionamento e se reconhecem como iniciantes no tema, estes foram classificados como profissionais juniores (Jr). Já os outros 7, possuem, em média, 4 anos de experiencia com projetos de descomissionamento e mais de 10 anos na área de $\mathrm{O} \& \mathrm{G}$, estes foram classificados como profissionais seniores $(\mathrm{Sr})$. Constatou-se coesão no julgamento de preferências entre critérios nos dois grupos, a medida que a frequência de posicionamento alcançada por cada critério não determinou nenhuma alteração no ordenamento, Tabela 3 .

Tabela 3 - Preferência entre critérios de avaliação das alternativas

\begin{tabular}{llcccccccccc} 
& \multicolumn{1}{c}{} & \multicolumn{1}{c}{$\mathbf{1}^{\mathbf{a}}$ ranking } & \multicolumn{2}{c}{$\mathbf{2}^{\mathbf{a}}$ ranking } & \multicolumn{2}{c}{$\mathbf{3}^{\mathbf{a}}$ ranking } & $\mathbf{4}^{\mathbf{a}}$ ranking & \multicolumn{2}{c}{$\mathbf{5}^{\mathbf{a}}$ ranking } \\
\cline { 3 - 13 } & $\mathrm{Jr}$ & $\mathrm{Sr}$ & $\mathrm{Jr}$ & $\mathrm{Sr}$ & $\mathrm{Jr}$ & $\mathrm{Sr}$ & $\mathrm{Jr}$ & $\mathrm{Sr}$ & $\mathrm{Jr}$ & $\mathrm{Sr}$ \\
\hline $\mathrm{C}_{5}$ & Segurança Operacional & 5 & 3 & 1 & 2 & 1 & 1 & 1 & 1 & 0 & 0 \\
$\mathrm{C}_{2}$ & Meio Ambiente & 2 & 2 & 3 & 3 & 2 & 2 & 1 & 0 & 0 & 0 \\
$\mathrm{C}_{1}$ & Técnico & 1 & 1 & 4 & 1 & 1 & 3 & 2 & 1 & 0 & 1 \\
$\mathrm{C}_{4}$ & Econômico & 0 & 1 & 0 & 0 & 2 & 1 & 2 & 3 & 4 & 2 \\
$\mathrm{C}_{3}$ & Social & 0 & 0 & 0 & 1 & 2 & 0 & 2 & 2 & 4 & 4 \\
\hline
\end{tabular}

Fonte: Elaboração própria.

Na Figura 6 e na Tabela 4 apresentam-se a consolidação da ordem de preferência dos 19 subcritérios de forma unificada, considerando todas as respostas dos formulários respondidos. 
Figura 6 - Radares de frequência de posição dos subcritérios de avaliação das alternativas por ordem de preferência
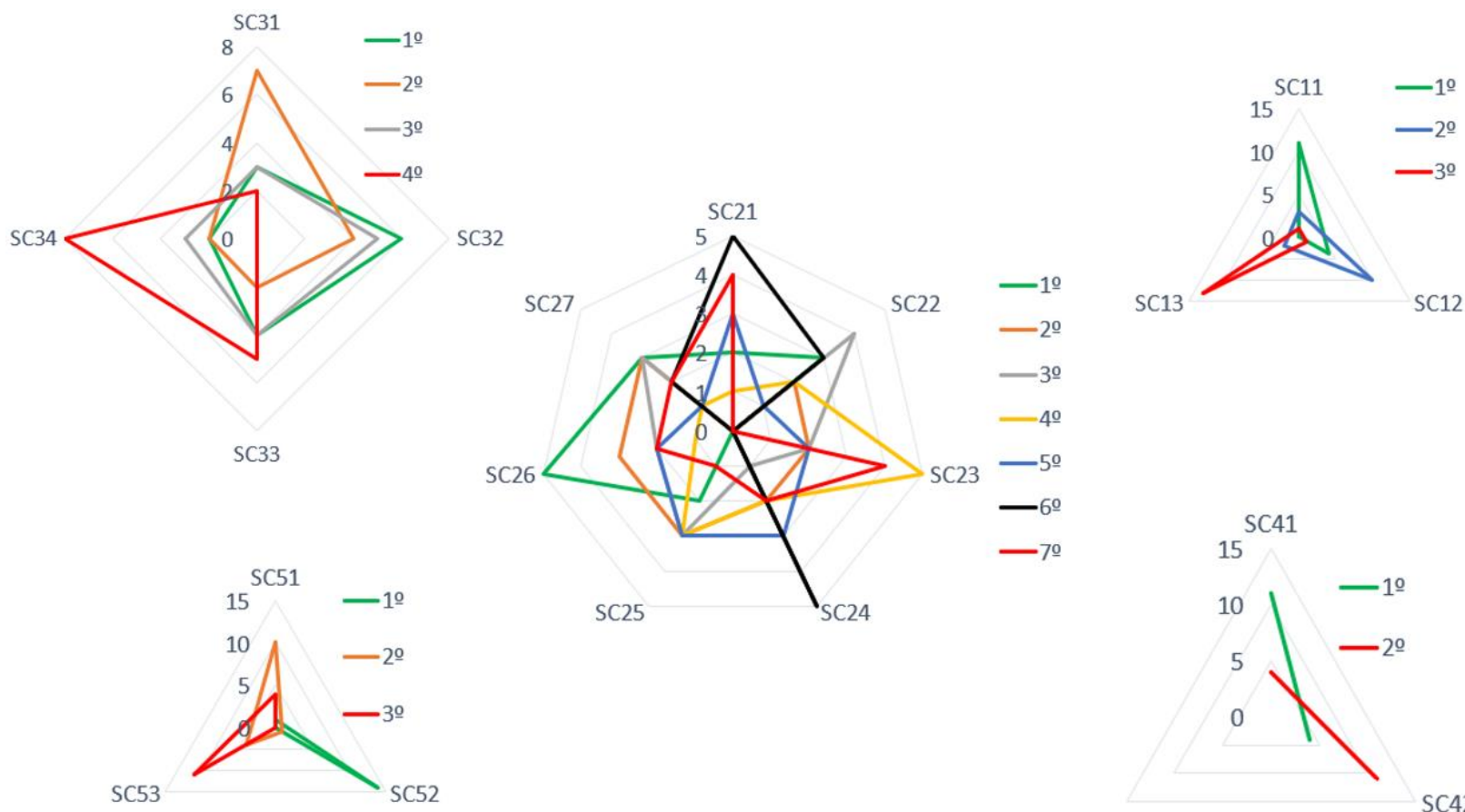

Fonte: Elaboração própria.

Tabela 4 - Número de ocorrências no ranking de preferência entre os subcritérios de avaliação das alternativas

\begin{tabular}{|c|c|c|c|c|c|c|c|c|}
\hline Critérios & Subcritérios & $1^{\circ}$ rank & $2^{\circ}$ rank & $3^{\circ}$ rank & $4^{\circ}$ rank & $5^{\circ}$ rank & $6^{\circ}$ rank & $7^{\circ}$ rank \\
\hline \multirow[t]{3}{*}{$\mathrm{C}_{1}$} & $\mathrm{SC}_{11}$ & 11 & 3 & 1 & - & - & - & - \\
\hline & $\mathrm{SC}_{12}$ & 4 & 10 & 1 & - & - & - & - \\
\hline & $\mathrm{SC}_{13}$ & 0 & 2 & 13 & - & - & - & - \\
\hline \multirow[t]{7}{*}{$\mathrm{C}_{2}$} & $\mathrm{SC}_{21}$ & 2 & 0 & 0 & 1 & 3 & 5 & 4 \\
\hline & $\mathrm{SC}_{22}$ & 3 & 2 & 4 & 2 & 1 & 3 & 0 \\
\hline & $\mathrm{SC}_{23}$ & 0 & 2 & 2 & 5 & 2 & 0 & 4 \\
\hline & $\mathrm{SC}_{24}$ & 0 & 2 & 1 & 2 & 3 & 5 & 2 \\
\hline & $\mathrm{SC}_{25}$ & 2 & 3 & 3 & 3 & 3 & 0 & 1 \\
\hline & $\mathrm{SC}_{26}$ & 5 & 3 & 2 & 1 & 2 & 0 & 2 \\
\hline & $\mathrm{SC}_{27}$ & 3 & 3 & 3 & 1 & 1 & 2 & 2 \\
\hline \multirow[t]{4}{*}{$\mathrm{C}_{3}$} & $\mathrm{SC}_{31}$ & 3 & 7 & 3 & 2 & - & - & - \\
\hline & $\mathrm{SC}_{32}$ & 6 & 4 & 5 & 0 & - & - & - \\
\hline & $\mathrm{SC}_{33}$ & 4 & 2 & 4 & 5 & - & - & - \\
\hline & $\mathrm{SC}_{34}$ & 2 & 2 & 3 & 8 & - & - & - \\
\hline \multirow[t]{2}{*}{$\mathrm{C}_{4}$} & $\mathrm{SC}_{41}$ & 11 & 4 & - & - & - & - & - \\
\hline & $\mathrm{SC}_{42}$ & 4 & 11 & - & - & - & - & - \\
\hline \multirow[t]{3}{*}{$\mathrm{C}_{5}$} & $\mathrm{SC}_{51}$ & 1 & 10 & 4 & - & - & - & - \\
\hline & $\mathrm{SC}_{52}$ & 14 & 1 & 0 & - & - & - & - \\
\hline & $\mathrm{SC}_{53}$ & 0 & 4 & 11 & - & - & - & - \\
\hline
\end{tabular}

Fonte: Elaboração própria. 
Sob a perspectiva da autodeclaração de experiência dos entrevistados percebe-se o mesmo alinhamento entre profissionais $\mathrm{Jr}$ e $\mathrm{Sr}$ no julgamento de preferências em todos os subcritérios, com exceção apenas nos que compõem o critério C2 - Meio Ambiente, ver Figura 7 e tabela 5.

Figura 7 - Radar de posição de frequência dos subcritérios SC2 Meio Ambiente - preferência por experiência profissional

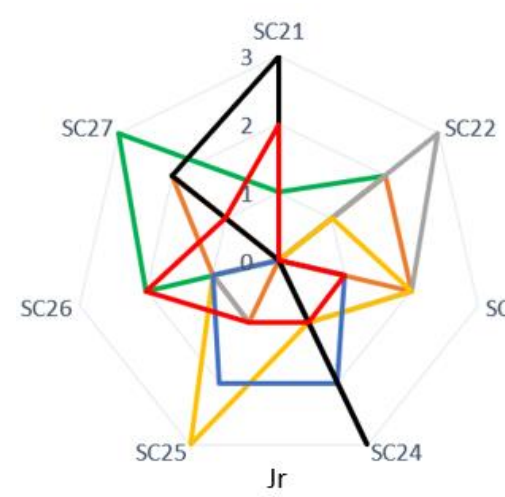

Fonte: Elaboração própria.

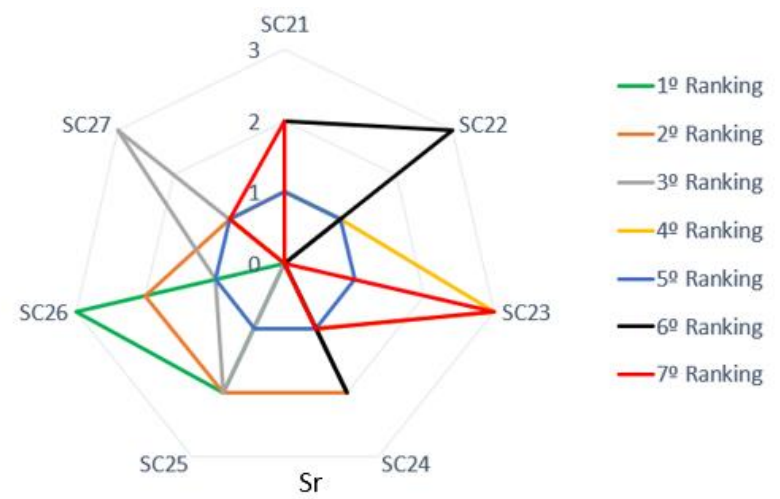

Tabela 5 - Alterações no Ranking para os subcritérios do critério Meio Ambiente

\begin{tabular}{llccc}
\hline $\mathrm{C}_{2}$ & Meio Ambiente & \multicolumn{3}{c}{ Ranking } \\
\hline & & $\mathrm{Jr}$ e $\mathrm{Sr}$ & $\mathrm{Jr}$ & $\mathrm{Sr}$ \\
$\mathrm{SC}_{21}$ & Emissão de $\mathrm{CO}_{2}$ & 7 & 7 & 6 \\
$\mathrm{SC}_{22}$ & Proporção de material reciclado & 2 & 1 & 4 \\
$\mathrm{SC}_{23}$ & Proporção de material descartado em aterro & 5 & 4 & 7 \\
$\mathrm{SC}_{24}$ & Consumo total de energia & 6 & 6 & 5 \\
$\mathrm{SC}_{25}$ & Perturbação do fundo do mar e / ou alteração do habitat & 3 & 5 & 2 \\
$\mathrm{SC}_{26}$ & Vazamentos acidentais & 1 & 3 & 1 \\
$\mathrm{SC}_{27}$ & Descarte de contaminantes & 4 & 2 & 3 \\
\hline
\end{tabular}

Fonte: Elaboração própria.

\subsection{Ponderação dos critérios e subcritérios}

Adotou-se a equação (1) na ordem de preferência dos critérios. Como não se percebeu alteração na ordem de preferência entre critérios, a Tabela 6 representa a ponderação para o caso global, que considera todas as respostas, bem como para profissionais Jr e Sr.

Tabela 6 - Ranking Order Centroid para os critérios

\begin{tabular}{lll}
\hline \multicolumn{2}{c}{ Peso dos Critérios } \\
\hline $\mathbf{C}_{5}$ & Segurança Operacional & 0,457 \\
\hline $\mathbf{C}_{2}$ & Meio Ambiente & 0,257 \\
$\mathbf{C}_{\mathbf{1}}$ & Técnico & 0,157 \\
$\mathbf{C}_{4}$ & Econômico & 0,090 \\
$\mathbf{C}_{3}$ & Social & 0,040 \\
\hline
\end{tabular}

Fonte: Elaboração própria. 
$\mathrm{Na}$ ordem de preferência dos subcritérios relativos a Meio Ambiente (Tabela 5), obtém-se ponderações diferentes para os agrupamentos de profissionais $\mathrm{Jr}$ e $\mathrm{Sr}$ após aplicação da equação (1), conforme Tabela 7.

Tabela 7 - Ranking Order Centroid para os subcritérios

\begin{tabular}{|c|c|c|c|c|c|c|c|c|c|c|c|}
\hline \multicolumn{2}{|c|}{$\mathrm{C}_{5}$} & \multicolumn{4}{|c|}{$\mathrm{C}_{2}$} & \multicolumn{2}{|c|}{$\mathrm{C}_{1}$} & \multicolumn{2}{|c|}{$\mathrm{C}_{4}$} & \multicolumn{2}{|c|}{$\mathrm{C}_{3}$} \\
\hline & & & Jr e $\mathrm{Sr}$ & $\mathrm{Jr}$ & $\mathrm{Sr}$ & & & & & & \\
\hline $\mathrm{SC}_{52}$ & 0,611 & $\mathrm{SC}_{26}$ & 0,370 & 0,156 & 0,370 & $\mathrm{SC}_{11}$ & 0,611 & $\mathrm{SC}_{41}$ & 0,75 & $\mathrm{SC}_{32}$ & 0,521 \\
\hline $\mathrm{SC}_{51}$ & 0,278 & $\mathrm{SC}_{22}$ & 0,228 & 0,370 & 0,109 & $\mathrm{SC}_{12}$ & 0,278 & $\mathrm{SC}_{42}$ & 0,25 & $\mathrm{SC}_{31}$ & 0,271 \\
\hline $\mathrm{SC}_{53}$ & 0,111 & $\mathrm{SC}_{25}$ & 0,156 & 0,073 & 0,228 & $\mathrm{SC}_{13}$ & 0,111 & & & $\mathrm{SC}_{33}$ & 0,146 \\
\hline & & $\mathrm{SC}_{27}$ & 0,109 & 0,228 & 0,156 & & & & & $\mathrm{SC}_{34}$ & 0,063 \\
\hline & & $\mathrm{SC}_{23}$ & 0,073 & 0,109 & 0,020 & & & & & & \\
\hline & & $\mathrm{SC}_{24}$ & 0,044 & 0,044 & 0,073 & & & & & & \\
\hline & & $\mathrm{SC}_{21}$ & 0,020 & 0,020 & 0,044 & & & & & & \\
\hline
\end{tabular}

Fonte: Elaboração própria.

\subsection{Aplicação dos pesos na Avaliação comparativa de descomissionamento no campo de Brent}

Situado no Mar do Norte e descoberto em 1971 o campo de Brent teve seu início de produção de petróleo iniciado em 1976. Formado por 4 plataformas, Alpha Jacket (estrutura fixa tipo jaqueta, Figura 3), Bravo, Charlie e Delta (tipo GBS - Gravity Based Structures), o campo de Brent teve o início do seu encerramento em 2011 marcado pela paralização na produção da plataforma Delta e, posteriormente, a interrupção total ocorreu em 2014 (Shell, 2017c).

Shell (2017b) adota um método de Análise Comparativa para avaliação de alternativas sob a perspectiva multicritério para descomissionamento de todo o campo de Brent, tubulações de interligação, estruturas submarinas e de superfície, poços etc. Neste artigo optou-se por analisar o efeito dos pesos dos critérios e subcritérios obtidos no item 4.2 na seleção de alternativas para descomissionamento da estrutura tipo jaqueta da unidade Brent Alpha Jacket. A análise comparativa adota como cenário base a ponderação linear de pesos para os cinco critérios, Técnico (20\%), Meio Ambiente (20\%), Social (20\%), Econômico (20\%) e Segurança Operacional (20\%) (Shell, 2017b). A distribuição dos pesos absolutos dos subcritérios consta na Tabela 8 , onde incorporou-se os pesos obtidos no item 4.2.

Tabela 8 - Peso dos subcritérios

\begin{tabular}{llcccc}
\hline Critério & & $\begin{array}{l}\text { Shell } \\
(\mathbf{2 0 1 7 a})\end{array}$ & $\mathbf{J r} \mathbf{e r}$ & $\mathbf{J r}$ & $\mathbf{S r}$ \\
\hline $\mathrm{SC}_{52}$ & $\begin{array}{l}\text { Exposição a risco da equipe de projeto } \\
\text { offshore }\end{array}$ & $6,70 \%$ & $27,90 \%$ & $27,90 \%$ & $27,90 \%$ \\
$\mathrm{SC}_{53}$ & $\begin{array}{l}\text { Exposição a riscos dos usuários das vias } \\
\text { marinhas }\end{array}$ & $6,70 \%$ & $5,07 \%$ & $5,07 \%$ & $5,07 \%$ \\
$\mathrm{SC}_{51}$ & $\begin{array}{l}\text { Exposição a risco da equipe de projeto } \\
\text { onshore }\end{array}$ & $6,70 \%$ & $12,70 \%$ & $12,70 \%$ & $12,70 \%$
\end{tabular}




\begin{tabular}{llcccc} 
& & & \multicolumn{3}{c}{ Conclusão } \\
$\mathrm{SC}_{25}, \mathrm{SC}_{26} \mathrm{e}$ & Impacto ambiental operacional & $5,00 \%$ & $16,30 \%$ & $11,72 \%$ & $19,36 \%$ \\
$\mathrm{SC}_{27}$ & & & & \\
$\mathrm{SC}_{22}$ e $\mathrm{SC}_{23}$ & Legado dos impactos ambientais & $5,00 \%$ & $7,71 \%$ & $12,29 \%$ & $3,31 \%$ \\
$\mathrm{SC}_{24}$ & Consumo total de energia & $5,00 \%$ & $1,13 \%$ & $1,13 \%$ & $1,87 \%$ \\
$\mathrm{SC}_{21}$ & Emissão de CO2 & $5,00 \%$ & $0,52 \%$ & $0,52 \%$ & $1,13 \%$ \\
$\mathrm{SC}_{11}, \mathrm{SC}_{12} \mathrm{e}$ & Viabilidade Técnica & $20,00 \%$ & $15,67 \%$ & $15,67 \%$ & $15,67 \%$ \\
$\mathrm{SC}_{13}$ & & & & & \\
\hline $\mathrm{SC}_{32}$ e $\mathrm{SC}_{34}$ & Impacto na pesca comercial & $6,70 \%$ & $2,33 \%$ & $2,33 \%$ & $2,33 \%$ \\
$\mathrm{SC}_{31}$ & Efeitos na geração de emprego & $6,70 \%$ & $1,08 \%$ & $1,08 \%$ & $1,08 \%$ \\
$\mathrm{SC}_{33}$ & Impacto nas comunidades & $6,70 \%$ & $0,58 \%$ & $0,58 \%$ & $0,58 \%$ \\
$\mathrm{SC}_{41}$ e $\mathrm{SC}_{42}$ & Econômico - Custo Total & $20,00 \%$ & $9,00 \%$ & $9,00 \%$ & $9,00 \%$ \\
\hline
\end{tabular}

Fonte: Elaboração própria.

Shell (2017a) apresenta 3 alternativas para o descomissionamento: remoção superior da estrutura da jaqueta com (1) remoção total da estrutura das estacas de fixação com corte externo, (2) remoção total da estrutura das estacas de fixação com corte interno e (3) abandono das estacas de fixação para manutenção ou formação de arrecifes. A performance de cada alternativa nos subcritérios apresentados encontra-se na Tabela 9.

Tabela 9 - Avaliação das alternativas

\begin{tabular}{|c|c|c|c|c|c|}
\hline Subcritério & & Unid. & $\begin{array}{l}\text { Alternativa } \\
\text { (1) }\end{array}$ & $\begin{array}{l}\text { Alternativa } \\
\text { (2) }\end{array}$ & $\begin{array}{c}\text { Alternativa } \\
\text { (3) }\end{array}$ \\
\hline $\mathrm{SC}_{52}$ & $\begin{array}{l}\text { Exposição a risco da equipe de } \\
\text { projeto offshore }\end{array}$ & $\mathrm{PPV}^{1}$ & 0,0209 & 0,0265 & 0,0025 \\
\hline $\mathrm{SC}_{53}$ & $\begin{array}{l}\text { Exposição a riscos dos usuários das } \\
\text { vias marinhas }\end{array}$ & $\mathrm{PPV}^{1}$ & 0 & 0 & 0,059 \\
\hline $\mathrm{SC}_{51}$ & $\begin{array}{l}\text { Exposição a risco da equipe de } \\
\text { projeto onshore }\end{array}$ & $\mathrm{PPV}^{1}$ & 0,0023 & 0,0023 & 0 \\
\hline $\begin{array}{l}\mathrm{SC}_{25}, \mathrm{SC}_{26} \mathrm{e} \\
\mathrm{SC}_{27}\end{array}$ & Impacto ambiental operacional & Nota & 0,71 & 0,94 & 1 \\
\hline $\mathrm{SC}_{22}$ e $\mathrm{SC}_{23}$ & Legado dos impactos ambientais & Nota & 1 & 1 & 0,7 \\
\hline $\mathrm{SC}_{24}$ & Consumo total de energia & $\mathrm{GJ}^{2}$ & 391.300 & 450.500 & 438.100 \\
\hline $\mathrm{SC}_{21}$ & Emissão de CO2 & Ton $\mathrm{CO}_{2}$ & 26.100 & 30.300 & 38.500 \\
\hline $\begin{array}{l}\mathrm{SC}_{11}, \mathrm{SC}_{12} \mathrm{e} \\
\mathrm{SC}_{13}\end{array}$ & Viabilidade Técnica & Nota & 0,7 & 0,6 & 1 \\
\hline $\mathrm{SC}_{32}$ e $\mathrm{SC}_{34}$ & Impacto na pesca comercial & $\mathfrak{£}^{3}$ & 1151976 & 1151976 & 0 \\
\hline $\mathrm{SC}_{31}$ & Efeitos na geração de emprego & $\mathrm{HH}^{4}$ & 233,2 & 238,1 & 13,6 \\
\hline $\mathrm{SC}_{33}$ & Impacto nas comunidades & Nota & 0,55 & 0,55 & 1 \\
\hline $\mathrm{SC}_{41}$ e SC 42 & Econômico - Custo Total & $£^{3} \mathrm{MM}$ & 58,53 & 59,77 & 3,41 \\
\hline
\end{tabular}

Nota. ${ }^{1} \mathrm{PPV}$ - Perda Potencial de Vida, ${ }^{2} \mathrm{GJ}$ - Gigajoule, ${ }^{3} £$ - libra esterlina, ${ }^{4} \mathrm{HH}$ - homem hora; Valores altos para os subcritérios avaliados como Nota indicam desempenho bom ou desejável.

Fonte: Adaptado de Shell (2017a)

Aplicou-se o método de Avaliação Comparativa do relatório Shell (2017b) com as ponderações da Tabela 8 e os valores de avaliação das alternativas obtidos da Tabela 9. Apresenta-se o resultado na Figura 8 , onde permanece a recomendação de decidir pela Alternativa 3, devido a melhor pontuação em todos os cenários. 
Figura 8 - Avaliação Comparativa de cada alternativa de descomissionamento da Plataforma Alpha e a contribuição de cada subcritério

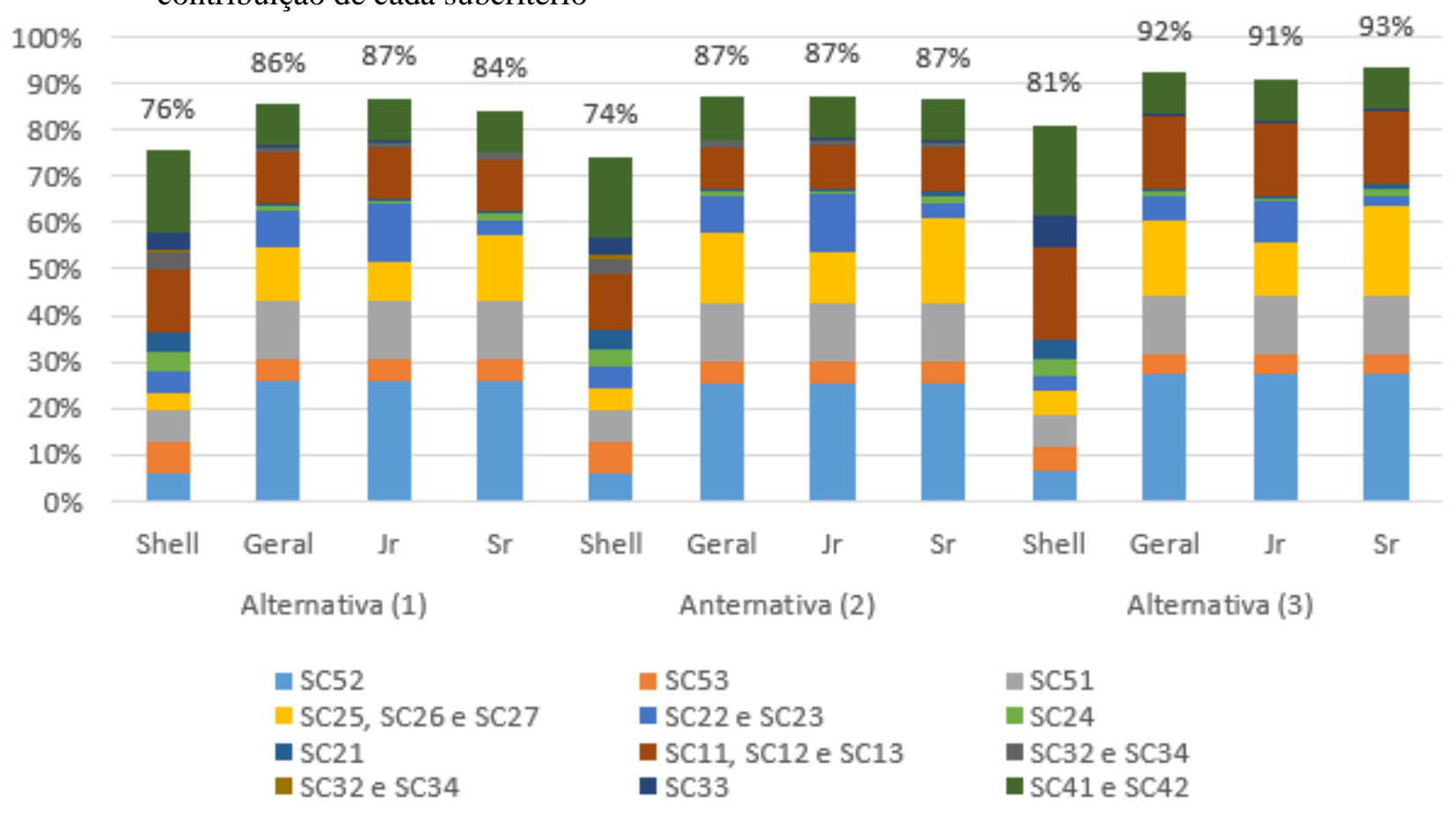

Fonte: Elaboração própria.

\subsection{Discussão}

Os resultados de preferências de ordenamento de critérios e subcritérios de avaliação de alternativas a projetos de descomissionamento demonstram o alinhamento de valores e a disseminação de conhecimento entre os membros da equipe de projeto participantes da pesquisa.

Em última análise, uma abordagem de descomissionamento flexível e holística será limitada pelo ambiente regulatório em que o projeto está inserido (Fowler et al., 2014). No Brasil, os projetos de descomissionamento de O\&G estão sujeitos ao atendimento de requisitos da resolução ANP $N^{\circ}$ 817. Entre os requisitos consta a elaboração de um Programa de Descomissionamento de Instalações (PDI), que contemple as informações, os projetos e os estudos necessários ao planejamento e à execução do descomissionamento de instalações. No PDI nenhum dos critérios, isoladamente, deverá ser considerado decisivo para a definição da alternativa (ANP, 2020b). No entanto, sob a ótica de instrumentos de incentivo de um agente regulador, acredita-se que a apresentação explícita da preferência de ordenamento de critérios, Tabela 6 , revela o compromisso prioritário da avaliação da equipe do projeto com critérios de segurança operacional, valorização da vida humana e do meio ambiente, o que possivelmente levaria a um parecer favorável a alternativa selecionada de forma mais célere.

Os pesos dos critérios e subcritérios (Tabela 7) oriundos da pesquisa de campo aplicados no relatório Shell (2017a, 2017b) possibilita aferir maior distância percentual entre a pior e a melhor alternativa no resultado 
da Avaliação Comparativa das alternativas, quando comparados com a distribuição linear de pesos entre critérios. No tocante ao processo de apoio a tomada de decisão a Alternativa (3) manteve-se com a melhor opção em qualquer um dos cenários de ponderação de pesos entre critérios e subcritérios apresentados. Já entre as demais alternativas, quando comparados os resultados de Shell (2017a, 2017b) com a adoção das ponderações de preferência do estudo exploratório aplicado, percebe-se a troca de posição entre as alternativas (1) e (2) devido ao maior peso dos critérios C5 Segurança operacional e C2 Meio Ambiente, assim como o menor peso do critério C4 Econômico.

As preferências no ordenamento dos critérios e subcritérios não podem ser extrapoladas para outros tipos de projetos de O\&G, visto que os projetos de descomissionamento proporcionam pouco ou nenhum retorno do investimento, principalmente quando comparados a outras fases do ciclo de vida de uma plataforma offshore (Eke et al., 2020).

\section{Considerações finais}

O propósito da modelagem AMD é, portanto, construir uma visão ou percepção das preferências do tomador de decisão consistente com um certo conjunto de suposições, de modo a dar uma orientação coerente ao tomador de decisão na busca pela solução preferida (Belton \& Stewart, 2002). Conhecer a hierarquia de preferência entre critérios e subcritérios utilizados na avaliação de projetos de descomissionamento permitiu constatar a sinergia e boa disseminação de conhecimentos entre membros de uma equipe de projetos com diferentes níveis de experiência profissional. Ao definir maior importância aos critérios de Segurança Operacional e Meio Ambiente, destaca-se a assimilação de valores éticos e pilares estratégicos empresariais por parte da equipe de projetos de descomissionamento entrevistada.

Projetos de descomissionamento em qualquer lugar do mundo estão inseridos em um contexto de rigorosas legislações (Fowler et al., 2014). No Brasil, além da ANP há órgãos fiscalizadores como o IBAMA (Instituto Brasileiro do Meio Ambiente e dos Recursos Naturais Renováveis), ICMBio (Instituto Chico Mendes de Conservação da Biodiversidade) ou instituições como a Marinha do Brasil, que podem impactar de maneira significativa a aprovação, realização e conclusão de um projeto de descomissionamento de plataformas offshore. Por possuírem instrumentos de incentivos diferentes, futuras abordagens podem concentrar-se em desenvolver estudos de forma a desnudar as estruturas de preferências, julgamentos de valor e práticas de negociação colaborativas destas outras partes interessadas.

\section{Referências}

ANP. (2020a). Boletim da Produção de Petróleo e Gás Natural. https://www.gov.br/anp/pt-br/centrais-deconteudo/publicacoes/boletinsanp/bmp/2020-09-boletim.pdf 
ANP. (2020b). RESOLUÇÃO No 817, DE 24

DE ABRIL DE 2020.

https://www.in.gov.br/en/web/dou//resolucao-n-817-de-24-de-abril-de-2020254001378

ANP. (2021). Painel Dinâmico de Descomissionamento. Agência Nacional Do Petróleo, Gás Natural e Biocombustíveis. https://www.gov.br/anp/pt-br/centrais-deconteudo/paineis-dinamicos-da-anp/paineldinamico-de-descomissionamento-deinstalacoes-de-exploracao-e-producao [acessado em 01 de fevereiro de 2021]

Barron, F. H., \& Barrett, B. E. (1996). The efficacy of SMARTER - Simple MultiAttribute Rating Technique Extended to Ranking. Acta Psychologica, 93(1-3), 23 36. https://doi.org/10.1016/00016918(96)00010-8

Belton, V., \& Stewart, T. J. (2002). Multiple Criteria Decision Analysis. In K. A. Publishers (Ed.), Multiple Criteria Decision Analysis. Springer US. https://doi.org/10.1007/978-1-4615-1495-4

Bull, A. S., \& Love, M. S. (2019). Worldwide oil and gas platform decommissioning: A review of practices and reefing options. Ocean and Coastal Management, 168(September 2018), 274-306. https://doi.org/10.1016/j.ocecoaman.2018.10 .024

Doumpos, M., \& Zopounidis, C. (2002). Multicriteria decision aid classification method. Kluwer Academic Publishers.

Edwards, W., \& Barron, F. H. (1994). SMARTS and SMARTER: Improved Simple Methods for Multiattribute Utility Measurement. In A Science of Decision Making (pp. 306-325).

https://doi.org/10.1093/acprof:oso/97801953 22989.003.0031.

Eke, E., Iyalla, I., Andrawus, J., \& Prabhu, R. (2020). Optimising offshore structures decommissioning - A multicriteria decisionapproach. Society of Petroleum Engineers - SPE Nigeria Annual International Conference and Exhibition
2020, NAIC 2020, 2018, 1-19.

https://doi.org/10.2118/203760-ms.

Ekins, P., Vanner, R., \& Firebrace, J. (2006). Decommissioning of offshore oil and gas facilities: A comparative assessment of different scenarios. Journal of

Environmental Management, 79(4), 420438.

https://doi.org/10.1016/j.jenvman.2005.08.0 23.

Fowler, A. M., Macreadie, P. I., Jones, D. O. B., \& Booth, D. J. (2014). A multi-criteria decision approach to decommissioning of offshore oil and gas infrastructure. Ocean and Coastal Management, 87, 20-29. https://doi.org/10.1016/j.ocecoaman.2013.10 .019 .

Gomes, L. F. A. M., \& Gomes, C. F. S. (2019). Princípios e Métodos para Tomada de Decisão - Enfoque Multicritério (6th ed.). Atlas.

Henrion, M., Bernstein, B., \& Swamy, S. (2015). A multi-attribute decision analysis for decommissioning offshore oil and gas platforms. Integrated Environmental Assessment and Management, 11(4), 594609. https://doi.org/10.1002/ieam.1693.

IEA. (2020). Fuels and technologies. https://www.iea.org/fuels-and-technologies [Acessado em 01 de fevereiro de 2021]

Investing. (2021). Petróleo Brent. https://br.investing.com/commodities/brentoil [acessado em 01 de fevereiro de 2021]

Kaiser, M. J. (2019). Decommissioning Forecasting and Operating Cost Estimation. Elsevier. https://doi.org/10.1016/C2018-002728-0.

Keeney, R. L., \& Raiffa, H. (1993). Decisions with Multiple Objectives Preferences and Value Tradeoffs (2nd ed.). Press Syndicate of the University of Cambridge.

Kueppers, L. M., Baer, P., Harte, J., Haya, B., Koteen, L. E., \& Smith, M. E. (2004). A decision matrix approach to evaluating the 
impacts of land-use activities undertaken to mitigate climate change: An editorial essay. Climatic Change, 63(3), 247-257. https://doi.org/10.1023/B:CLIM.000001859 0.49917.50.

Marfatia, F. (2019). Digitally Transforming Front End Decommissioning Planning. SPE Symposium: Decommissioning and Abandonment, December, 3-4. https://doi.org/10.2118/199218-MS.

Martins, I., Bahiense, L., Infante, C. E. D., \& Arruda, E. F. (2020). Dimensionality reduction for multi-criteria problems: An application to the decommissioning of oil and gas installations. Expert Systems with Applications, 148. https://doi.org/10.1016/j.eswa.2020.113236.

Martins, I. D., Moraes, F. F., Távora, G., Soares, H. L. F., Infante, C. E., Arruda, E. F., Bahiense, L., Caprace, J., \& Lourenço, M. I. (2020). A review of the multicriteria decision analysis applied to oil and gas decommissioning problems. Ocean and Coastal Management, 184(October 2019). https://doi.org/10.1016/j.ocecoaman.2019.10 5000 .

OGUK. (2015). Guidelines for Comparative Assessment in Decommissioning Programmes (UK Oil and Gas Industry Association Limited

OGUK. (2020). Decommissioning insight 2020.

https://oilandgasuk.co.uk/product/decommissio ning-insight-report/ [acessado em 01 de fevereiro de 2021]

Repsol. (2017). UKCS Decommissioning Programme.https://www.repsol.no/imagenes /repsolpornr/no/decommissioning_program me_tcm89-119886.pdf [acessado em $01 \mathrm{de}$ fevereiro de 2021]

Saaty, T. L. (2008). Decision making with the analytic hierarchy process. Int. J. Services Sciences, 1(1), 83-98. https://doi.org/10.1016/03050483(87)90016-8.
Schroeder, D. M., \& Love, M. S. (2004). Ecological and political issues surrounding decommissioning of offshore oil facilities in the Southern California Bight. Ocean and Coastal Management, 47(1-2). https://doi.org/10.1016/j.ocecoaman.2004.03 .002 .

Shell. (2017a). Brent Alpha Jacket Decommissioning Technical Document (Issue February).

Shell. (2017b). Brent Field Decomissioning: Comparative Assessment Procedure. https://www.shell.co.uk/sustainability/deco mmissioning/brent-fielddecommissioning/brent-fielddecommissioningprogramme/_jcr_content/par/tabbedcontent/t ab_1385449832/textimage.stream/14864718 99349/14e9687bafecccbbd0a211b8f518c14 545018f04/brent-decom-comp [acessado em 01 de fevereiro de 2021]

Shell. (2017c). The Brent History. https://www.shell.co.uk/sustainability/deco mmissioning/brent-fielddecommissioning/the-brent-story.html [acessado em 01 de fevereiro de 2021]

Speight, J. G. (2015). Offshore Platforms. In Subsea and Deepwater Oil and Gas Science and Technology. https://doi.org/10.1016/b978-1-85617-5586.00003-9

STATISTA, 2018. Number of offshore rigs worldwide as of January 2018 by region [online]. New York, NY. Disponível em: https://www.statista.com/statistics/279100/n umber-of-offshore-rigs-worldwide-byregion/ [acessado em 01 de fevereiro de 2021].

UN. (1958). United Nations Convention on the Continental Shelf. Treaty Series, 499(June 1964), 311.

https://legal.un.org/ilc/texts/instruments/engl ish/conventions/8_1_1958_continental_shelf .pdf / [acessado em 01 de fevereiro de 2021]

UN. (1995). United Nations Convention on the Law of the Sea. Ocean Development and 
International Law, 26(4), 391-412.

https://doi.org/10.1080/00908329509546068

Vergara, S. C. (2016). Administração, Projetos

e Relatórios de Pesquisa em Administração; 6th ed.). Atlas. 